ISSN 2519-7398 (Versión electrónica)

DOI: http://dx.doi.org/10.21704/ac.v78i2.1067

(C) Universidad Nacional Agraria La Molina, Lima - Perú

\title{
Obtención y caracterización de un hidrolizado de colágeno purificado producido mediante el uso de la enzima delvolase
}

\author{
Obtaining and characterizing a purified collagen hydrolyzize produced by the use of the enzyme \\ delvolase
}

Tito Eduardo Lleren Daza ${ }^{1 *}$; Wendy Patricia Rodríguez Torres ${ }^{2}$.

*Autor de correspondencia

\section{Resumen}

El objetivo de la investigación fue obtener un hidrolizado proteico por vía enzimática, a partir de colágeno purificado de pescado, mediante el uso de la proteasa Delvolase. Además, se buscó caracterizar el hidrolizado de colágeno purificado de pescado, obtenido con los parámetros más eficientes, mediante la determinación de algunas de sus propiedades funcionales. La hidrólisis fue catalizada por una proteasa comercial (Delvolase ${ }^{\circledR}$ ), se optimizó las condiciones de hidrólisis con el uso de la metodología de superficie de respuesta (MSR). El modelo fue propuesto con respecto a los efectos de la temperatura $(T)$, tiempo $(t)$ y enzima $(E)$, en rangos de $T: 55-65{ }^{\circ} \mathrm{C}, t: 2 \mathrm{~h}-6 \mathrm{~h}, E$ : $0,03 \%-$ $0,15 \%$ respectivamente, encontrándose un óptimo grado de hidrólisis $(\mathrm{GH})$ de $12,59 \%$ para un tiempo de 2 horas, a una temperatura óptima de $64,97{ }^{\circ} \mathrm{C}$. El hidrolizado de colágeno purificado optimizado, se caracterizó por presentar en base húmeda $12,55 \%$ de proteína, $0,32 \%$ de grasa y $1 \%$ de ceniza. Así mismo, se caracterizó por poseer una buena capacidad para gelificar, formar espuma, emulsificar y absorber aceite. La hidrólisis proteica del colágeno de pescado es una alternativa recomendable para la elaboración de nuevos productos biotecnológicos.

Palabras claves: hidrolizado de colágeno, superficie de respuesta, proteasa, caracterización del hidrolizado, propiedades funcionales.

Abstract
The objective of the research was to obtain a protein hydrolyzate by enzymatic route, from purified fish collagen, with the use of the protease Delvolase. In addition, we sought to characterize the purified fish collagen hydrolyzate, obtained with the most efficient parameters, by determining some of its functional properties. The hydrolysis was catalyzed by a commercial protease (Delvolase $\left.{ }^{\circledR}\right)$, hydrolytic conditions were optimized with the use of surface response methodology (MSR). The model was proposed in relation to effects of temperature $(T)$, time $(t)$ and enzyme $(E)$, in ranges of $T: 55-65$ ${ }^{\circ} \mathrm{C}, t: 2 \mathrm{~h}-6 \mathrm{~h}, E: 0,03 \%-0,15 \%$ respectively. The optimal hidrolysis degree (HD) was $12,59 \%$ for 2 hours in a slightly alkaline way. The fish collagen hidrolyzate obtained with optimal parameters showed in wet basis $12,55 \%$ of protein, $0,32 \%$ fat, $1 \%$ of ashes. Likewise, it was characterized by having a good ability to gel, foam, emulsify and absorb oil. Protein hydrolysis of fish collagen is an advisable alternative for the development of new biotechnological products.

Keywords: collagen hydrolysate, response surface, protease, characterization of hydrolysate, functional properties.

\section{Introducción}

Los residuos de pescado son considerados comercialmente la parte no comestible del pescado (cabeza, agallas, vísceras, espinazo y cola, piel, orejetas sangre y otros). Fernández (2001) afirma que los residuos constituyen alrededor del 50\% de la materia prima, mientras que Pizardi (1999) citado por Fernández (2001), menciona que los residuos producidos por la industria pesquera fluctúan entre $38 \%$ y $70 \%$. Por otra parte, las características de los residuos dependen de la proporción de los componentes físicos y químicos. Estos residuos pueden estar constituidos por los componentes de una o varias especies de pescado (Fernández, 2001).

Para las industrias tradicionales de conservas, curados y otras industrias de procesamiento de recursos hidrobiológicos, los residuos sólidos que se producen crean verdaderos problemas de eliminación. El uso adecuado de estos desechos puede constituir un aporte de alto valor biológico y un incentivo económico importante a las industrias que lo producen.

\footnotetext{
${ }^{1}$ Departamento Académico de Industrias Pesqueras, Facultad de Pesquería, Universidad Nacional Agraria La Molina, Apartado postal 12-056 - La Molina, Lima, Perú. Email: telld@lamolina.edu.pe

${ }^{2}$ Tesista, Universidad Nacional Araria La Molina, Lima, Perú. Email:wp.rodriguezt@gmail.com
} 
Una alternativa de uso de estos desechos es utilizarlos para producir hidrolizados, ya que son una importante fuente de nutrientes. Al ser hidrolizados estos desechos, ocurre una disminución en el tamaño de péptidos, modificando las características funcionales de la proteína, por lo que mejorará su calidad (Kristinsson y Rasco, 2000).

La presente investigación se realiza con la finalidad de plantear una alternativa para el aprovechamiento de un colágeno de pescado, el cual al ser hidrolizado mediante el uso de una enzima proteasa se modificarán sus propiedades funcionales, diversificando sus posibles usos en la industria.

Así mismo, se buscó proponer este producto como fuente potencial de proteína para la aplicación en bienes de consumo humano que otorgue alguna mejora en la salud y bienestar corporal de las personas, o pueda ejercer un efecto por vías distintas a la ingesta, como puede ser la absorción por la piel o la inyección en la zona en que se desea ejerza su acción. En tales casos, estaríamos considerándolo para sus aplicaciones cosméticas, farmacológicas o médicas (Anfaco, 2012).

\section{Materiales y métodos}

\section{Materia prima}

Se obtuvo un colágeno purificado de pescado obtenido a partir de la vejiga natatoria de bagre (Rhamdia sapo), para obtener el hidrolizado de colágeno mediante el proceso enzimático. El material fue adquirido de una empresa comercializadora de aditivos industriales en la ciudad de Lima.

\section{Enzima utilizada}

Se utilizó Delvolase ${ }^{\circledR}$, una enzima proteasa alcalina con actividad endopeptidasa, cuyas condiciones de máxima actividad son, $\mathrm{pH} 6,0$ y T $65^{\circ} \mathrm{C}$.

\section{Obtención de la muestra y tratamientos de hidrólisis}

La preparación de la muestra consistió en hidratar el colágeno purificado con agua destilada temperada en una relación de 1:8, según pruebas preliminares realizadas. Se ajustó el $\mathrm{pH}$ de dicha mezcla con $\mathrm{NaOH}$ o $\mathrm{HCl}$ hasta encontrar en el rango óptimo de acción de la enzima, pH óptimo entre 9 y 10, según $M S D S$ (Material Safety Data Sheet) de la enzima utilizada en la presente investigación. La suspensión preparada con $\mathrm{pH}$ ajustado al rango óptimo de acción de la enzima (entre 9 y 10), fue homogeneizada en un vaso de precipitación mediante agitación, por espacio de 5 minutos, luego fue trasladada a tubos de ensayo, donde se añadió la cantidad de enzima establecida para cada tratamiento, posteriormente, fueron sometidas a temperaturas controladas correspondientes para cada tratamiento, mediante baño María. Finalmente, el hidrolizado fue centrifugado con el fin de separar las fracciones proteicas solubles e insolubles, utilizando para ello un papel filtro. En la presente investigación se utilizó la fracción soluble.

\section{Condiciones de hidrólisis}

Se realizaron ensayos para determinar las condiciones más adecuadas para la obtención del hidrolizado proteico de colágeno purificado de pescado, con el fin de evaluar el comportamiento de la enzima Delvolase $(0,03 \%$ y $0,15 \%)$, la temperatura $\left(55^{\circ} \mathrm{C}\right.$ y $\left.65^{\circ} \mathrm{C}\right)$ y el tiempo $(2 \mathrm{~h}$ y $6 \mathrm{~h}$ ) en el proceso de hidrólisis. Para el experimento se propusieron tres variables respuestas: grado de hidrólisis (GH), porcentaje de proteína y capacidad de retención de agua (CRA). Las condiciones de hidrólisis se basaron en las combinaciones establecidas por el programa DesignExpert 7.0, utilizando el método de superficie de respuesta, mediante el cual se obtuvieron 20 puntos individuales para ser empleados en el experimento. Se determinaron las mejores condiciones de hidrólisis, tomando el tratamiento con mejor resultado, para someterlo a un ajuste. Finalmente se evaluaron las características en el hidrolizado proteico de colágeno de pescado obtenido con los parámetros más eficientes.

\section{Ensayos para la determinación de la actividad enzimática}

Para la determinación de la actividad enzimática se consideraron las determinaciones de: valor de $\mathrm{pH}$ (Castañón, 2009), grado de hidrólisis (Dumay et al., (2004 y Pandia, 2011), cuantificación de proteína mediante la reacción Biuret (Gornall et al., (1949) y capacidad de retención de agua (Rodríguez-Ambriz et al., 2005).

\section{Caracterización organoléptica}

La caracterización organoléptica del Hidrolizado Proteico de Pescado se realizó de acuerdo a Fernández (2001). Para la evaluación de la calidad sensorial del producto final se consideraron los siguientes atributos: olor, color y consistencia.

\section{Caracterización del hidrolizado}

Al hidrolizado proteico de colágeno de pescado obtenido con los parámetros más eficientes se le caracterizó mediante la determinación de sus propiedades funcionales y su composición físico - química.

Para la determinación de sus propiedades funcionales, se realizaron los siguientes análisis: capacidad de emulsificación (Yu et al., 2007), capacidad de formación de espuma (Yu et al., 2007), capacidad de absorción de aceite (Lin et al., 1974), capacidad de gelificación (Olivares, 2009). Así mismo, para la determinación de la composición físico - química de la fracción soluble del hidrolizado obtenido, se le determinaron los siguientes parámetros: pH (Castañón, 2009), composición química proximal (AOAC, 1990): humedad (\%) por el método gravimétrico, proteína total (\%) por el método Kjeldahl, grasa cruda (\%) por el método Soxhlet, cenizas $(\%)$ por el método gravimétrico.

\section{Análisis estadísticos}

Los resultados obtenidos en laboratorio se evaluaron mediante el uso del programa Design Expert 7.0, utilizando 
el análisis de varianza (ANOVA), mediante el método de superficie de respuesta. Con el uso del programa Minitab 2015, se realizó la comparación de medias, analizando las diferencias significativas entre los valores prácticos y teóricos obtenidos en el presente estudio.

\section{Resultados y discusiones}

\section{Caracterización de la materia prima}

El componente químico mayoritario en el hidrolizado de colágeno de pescado fueron las proteínas $(\mathrm{Nx} 6,25)$, con un $60,0 \%$, mientras que el contenido de humedad fue de un $12 \%$. Estos valores son similares a los concentrados proteicos elaborados en base a subproductos de pescado, los cuales contienen alto porcentaje de proteínas (Woo et al., 2008). El contenido de grasa fue de 1,3\%, este parámetro podría resultar importante por el posible contenido de ácidos grasos $\omega$-3 que pudiera contener, y el contenido de ceniza fue de $1,0 \%$. Finalmente, la materia prima presentó un valor de $\mathrm{pH}$ de 3,0 resultado que está relacionado con la presentación comercial del colágeno de pescado, que es un mezclado con ácido cítrico que facilita la disolución y acelera la floculación y sedimentación de coloides solubles en suspensión (Hidalgo, 2014).
Evaluación del efecto que ejercen las variables independientes sobre las variables respuestas (GH, CRA y proteína)

En la Tabla 1, se presentan las combinaciones de factores (tiempo, temperatura y concentración de enzima) y las variables respuesta por cada tratamiento $(\mathrm{GH}$, proteína y CRA), empleados en el diseño compuesto central. Así mismo; en la Tabla 2 se presentan los valores obtenidos del diseño compuesto central de la hidrólisis de colágeno de pescado purificado, correspondiente al total de las combinaciones de los factores en estudio. Se evidencian temperaturas fluctuantes entre 55 y $65^{\circ} \mathrm{C}$, presentando una media de $60{ }^{\circ} \mathrm{C}$ con una tendencia a variar por debajo o por encima de dicha temperatura en $4,13{ }^{\circ} \mathrm{C}$. Con respecto al factor tiempo, fluctuó entre 2,2 y $5,8 \mathrm{~h}$; presentando una media de $4 \mathrm{~h}$ con una tendencia a variar de 1,48 h por debajo o por encima del mencionado tiempo. El factor concentración de la enzima presentó una fluctuación entre 0,05 y 0,13 por ciento una media de 0,09 por ciento y una desviación estándar de 0,03 por ciento lo que nos indica que tuvo una tendencia a variar de 0,03 por ciento por debajo o por encima del valor de la media.

Taba 1: Combinaciones de factores y las variables respuesta por tratamientos

\begin{tabular}{ccccccc}
\hline \multirow{2}{*}{ Tratamiento } & $\begin{array}{c}\text { Factor 1 } \\
\text { A: Temp. }\left({ }^{\circ} \mathrm{C}\right)\end{array}$ & $\begin{array}{c}\text { Factor } 2 \\
\text { B: Tiempo }(\mathrm{h})\end{array}$ & $\begin{array}{c}\text { Factor 3 C: } \\
\text { Enzima }(\%)\end{array}$ & $\begin{array}{c}\text { Var Resp } 1 \mathrm{GH} \\
(\%)\end{array}$ & $\begin{array}{c}\text { Var Resp 2 } \\
\text { Proteína }(\%)\end{array}$ & $\begin{array}{c}\text { Var Resp 3 CRA } \\
(\mathrm{ml} \mathrm{H} \text { O/g m) }\end{array}$ \\
\hline 1 & 55 & 2,2 & 0,05 & 7,09 & 7,41 & 3,6 \\
2 & 65 & 2,2 & 0,05 & 13,69 & 12,63 & 4 \\
3 & 55 & 5,8 & 0,05 & 14,03 & 7,92 & 0,8 \\
4 & 65 & 5,8 & 0,05 & 12,53 & 11,68 & 3,8 \\
5 & 55 & 2,2 & 0,13 & 13,78 & 8,19 & 4 \\
6 & 65 & 2,2 & 0,13 & 13,33 & 12,69 & 0,8 \\
7 & 55 & 5,8 & 0,13 & 11,47 & 8,68 & 0,6 \\
8 & 65 & 5,8 & 0,13 & 9,25 & 11,17 & 1,2 \\
9 & 51,59 & 4 & 0,09 & 10,14 & 6,31 & 3,6 \\
10 & 68,41 & 4 & 0,09 & 9,96 & 10,06 & 4 \\
11 & 60 & 0,97 & 0,09 & 9,65 & 13,27 & 1 \\
12 & 60 & 7,03 & 0,09 & 13,75 & 7,80 & 1 \\
13 & 60 & 4 & 0,03 & 6,75 & 5,32 & 1,2 \\
14 & 60 & 4 & 0,16 & 6,87 & 6,61 & 0,6 \\
15 & 60 & 4 & 0,09 & 6,64 & 5,26 & 4 \\
16 & 60 & 4 & 0,09 & 6,13 & 5,49 & 4 \\
17 & 60 & 4 & 0,09 & 6,79 & 5,51 & 4,12 \\
18 & 60 & 4 & 0,09 & 6,47 & 5,52 & 4 \\
19 & 60 & 4 & 0,09 & 6,62 & 5,49 & 3,9 \\
\hline
\end{tabular}

Tabla 2. Resultados del diseño estadístico de los factores

\begin{tabular}{ccccccc}
\hline Factor & Nombre & Unidad & Menor nivel & Mayor nivel & Media & Desv. Estándar \\
\hline $\mathrm{A}$ & Temperatura & ${ }^{\circ} \mathrm{C}$ & 55 & 65 & 60 & 4,13 \\
$\mathrm{~B}$ & Tiempo & $\mathrm{H}$ & 2,2 & 5,8 & 4 & 1,48 \\
$\mathrm{C}$ & Enzima & $\%$ & 0,05 & 0,13 & 0,09 & 0,03 \\
\hline
\end{tabular}




\section{Diferencias entre los factores de los tratamientos}

El análisis de superficie de respuesta para el grado de hidrólisis del colágeno de pescado purificado en función al tiempo y la temperatura, concentración de enzima y temperatura, concentración de enzima y tiempo, se presenta en la Figura 1. Para los tres casos se puede observar una superficie de respuesta en forma de valle, lo que se explica debido a que la temperatura, el tiempo y la enzima, cada uno por separado no influyeron significativamente sobre el grado de hidrólisis, con un $p$-value menor a 0,05 , las temperaturas fluctúan entre 55 y $65^{\circ} \mathrm{C}$, presentando una media de $60{ }^{\circ} \mathrm{C}$ con una tendencia a variar por debajo o por encima de dicha temperatura en $4,13^{\circ} \mathrm{C}$. Sin embargo, la interacción entre tiempo-concentración de enzima si influyeron significativamente en el grado de hidrólisis ( $p$-value $=0,0249)$, El factor tiempo fluctuó entre 2,2 y 5,8 $\mathrm{h}$; presentando una media de $4 \mathrm{~h}$ con una tendencia a variar de 1,48 h por debajo o por encima del mencionado tiempo. Así mismo, la interacción entre temperatura-concentración de enzima no influyeron significativamente en la variación del grado de hidrólisis (p-value mayor a 0,05). El factor concentración de la enzima presentó una fluctuación entre 0,05 y 0,13 por ciento una media de 0,09 por ciento y una desviación estándar de 0,03 por ciento lo que nos indica que tuvo una tendencia a variar de 0,03 por ciento por debajo o por encima del valor de la media. Con respecto a la interacción temperatura-tiempo se encontraron en el límite o similar al valor de $\alpha=0,05$, lo que indica que su interacción podría influenciar significativamente sobre la variable respuesta grado de hidrólisis.

Por otro lado, la variable respuesta grado de hidrólisis, presentó fluctuaciones de valores entre 6,13 y14,02 por ciento, cuyo porcentaje promedio fue de 9,58 con una tendencia a variar por debajo o por encima de dicho porcentaje en $2,96 \%$.

Así mismo, la variable respuesta proteína presentó fluctuaciones de valores entre 5,26 y 13,27 por ciento, cuyo promedio fue de 8,12 por ciento, presentando una tendencia a variar de 2,75 por ciento por debajo o por encima del promedio.

Por último, los valores de la variable respuesta capacidad de retención de agua, fluctuaron entre 0,6 y 4,12 $\mathrm{ml} \mathrm{H}_{2} \mathrm{O} / \mathrm{g}$

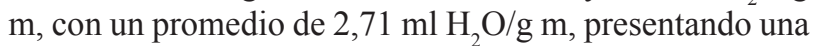
tendencia a variar de $1,49 \mathrm{ml} \mathrm{H}_{2} \mathrm{O} / \mathrm{g} \mathrm{m}$ por debajo o por encima del promedio.

Con respecto al coeficiente de variabilidad para las tres variables resultaron ser menor al 50 por ciento (GH: 17,02 por ciento, proteína: 16,71 por ciento, CRA: 23,44 por ciento), lo que indica una baja dispersión de los datos y en consecuencia una buena representatividad de la media (Gutiérrez, 2012).
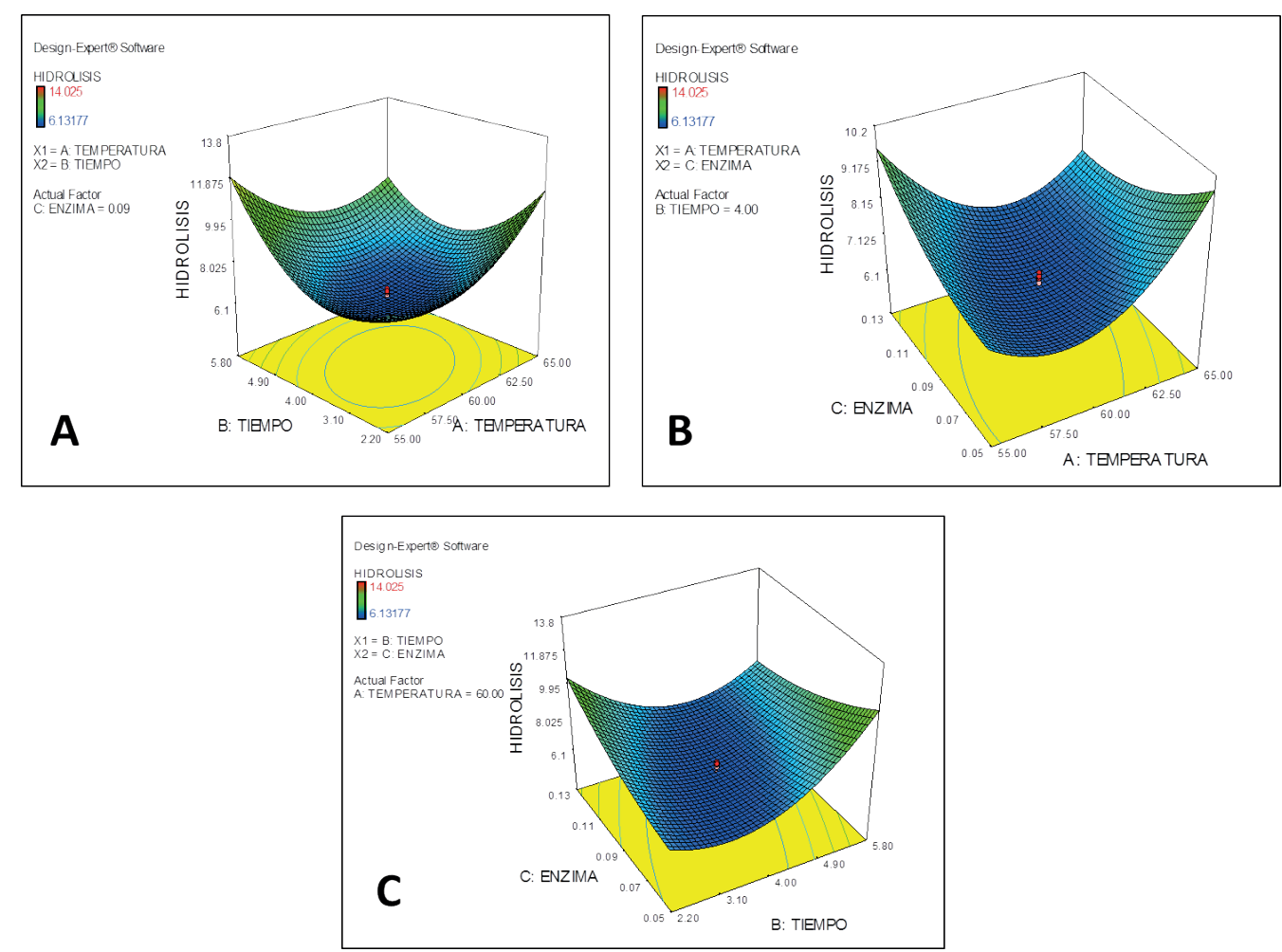

Figura 1. Superficie de respuesta del grado de hidrólisis del colágeno de pescado purificado: (A) en funcion a las variables tiempo y temperatura; (B) en funcion a las variables concentración de enzima y temperatura. (C) en funcion a las variables concentración de enzima y tiempo. 
El análisis de superficie de respuesta para la proteína del colágeno purificado de pescado en función del tiempo y temperatura, concentración de enzima y temperatura, concentración de enzima y tiempo se presentan en la Figura 2. Para los tres casos se puede observar una superficie de respuesta en forma de valle, esto se explica debido a que existieron evidencias significativas de que la temperatura influyó significativamente en la variación de la proteína $(p$-value $=0,0013)$, no obstante, el tiempo y la enzima cada uno por separado no influyeron significativamente en la variación de la misma, debido a que el $p$-value fue menor a 0,05. Así mismo, la interacción entre tiempo-concentración de enzima, temperaturaconcentración de enzima y temperatura-tiempo, no influyeron significativamente sobre la variable respuesta proteína ( $p$-value mayor a 0,05$)$. significativamente en la capacidad de retención de agua, ya que poseen un $p$-value menor a 0,05 . Sin embargo, la interacción entre tiempo-concentración de enzima no influyeron significativamente en la capacidad de retención de agua ( $p$-value $=1,00)$. Por otro lado, a un nivel de confianza de 95,0 por ciento, la interacción entre temperatura-concentración de enzima y temperaturatiempo influyeron significativamente en la capacidad de retención de agua ya que poseen un p-value menor a 0,05 . Así mismo, el valor del coeficiente de determinación para el modelo ajustado de la variable CRA fue de 90,92 por ciento, lo que indica que el modelo explica el 90,92 por ciento de la variación total.
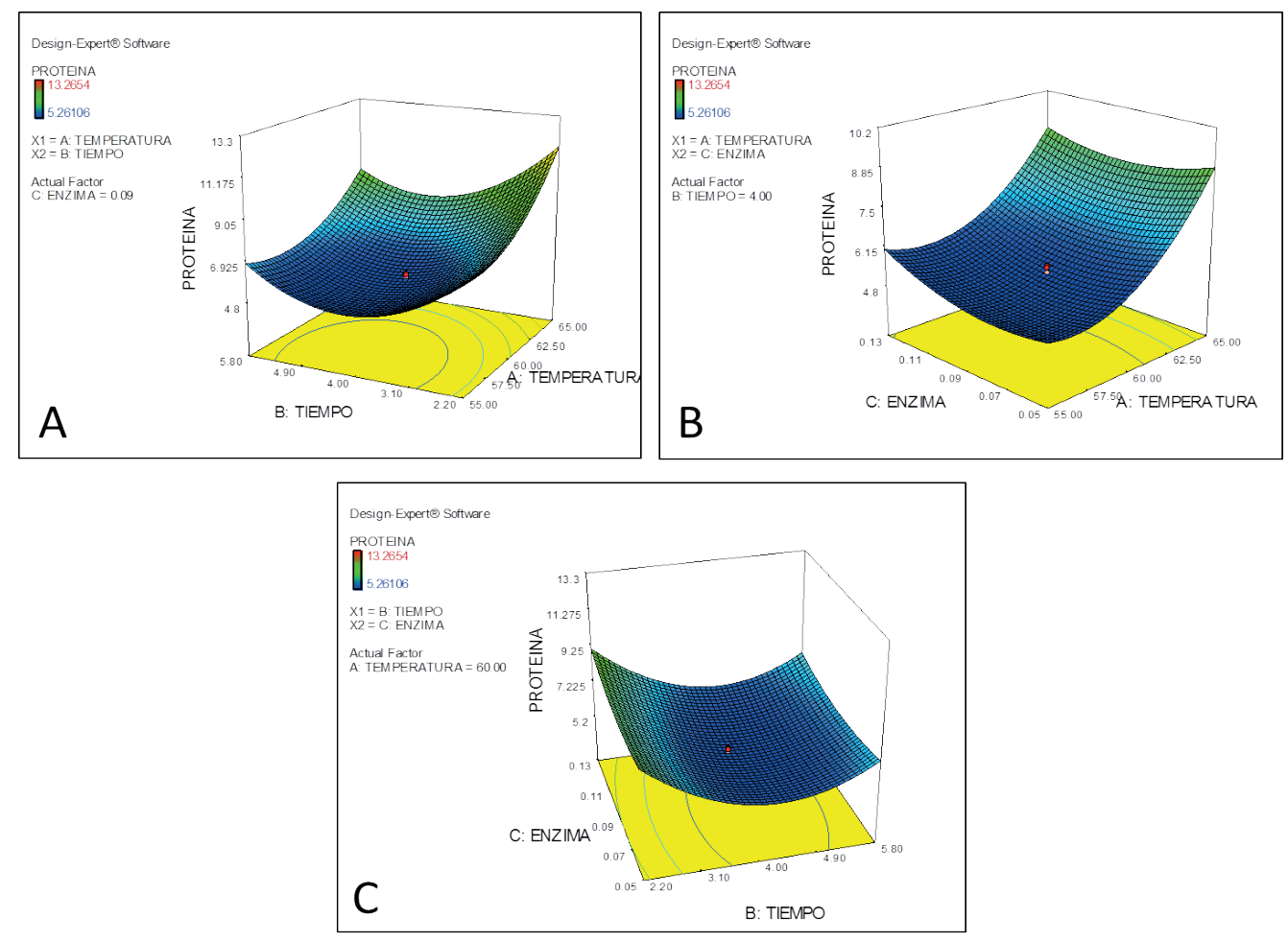

Figura 2. Superficie de respuesta de proteínas del colágeno de pescado (A) en función a las variables temperatura y tiempo; (B) en función a las variables concentración de enzima y temperatura; (C) en función a las variables concentración de enzima y tiempo

El análisis de superficie de respuesta para la capacidad de retención de agua del colágeno de pescado en función a la temperatura-tiempo, concentración de enzima-temperatura, concentración de enzima-tiempo, se presenta en la Figura 3. Para los tres casos se puede observar una superficie de respuesta en forma de montaña, esto nos conlleva a deducir que no existieron evidencias significativas de que la temperatura influyera significativamente en la capacidad de retención de agua, debido a que posee un $p$-value mayor a 0,05 . No obstante, el tiempo y la enzima cada uno por separado si influyeron
Finalmente, para realizar el análisis de superficie de respuesta para las condiciones del punto óptimo teórico del colágeno de pescado en función a la temperatura $65^{\circ} \mathrm{C}$ y el tiempo de $2,2 \mathrm{~h}$ se presenta en la Figura 4, donde se puede observar que el punto óptimo se encuentra en la parte superior de la gráfica ascendente, con una deseabilidad de 0,92 .

El ajuste de las condiciones de hidrólisis para la optimización del GH se realizó en función al tiempo y grado de hidrólisis. Esta consistió en mantener los valores de temperatura y enzima dentro de los rangos 
estudiados debido a que no influyen significativamente en el grado de hidrólisis, así mismo se propuso optimizar el tiempo, reducíendolo al mínimo del valor estudiado, con el fin de disminuir costos. Por otro lado se optimizó el GH al máximo valor empelado. Con la ayuda del programa Design Expert, escogiendo la combinación que tuviera mayor deseabilidad $(0,92)$, según el método de deseabilidad donde cero representa una respuesta no deseable y uno representa la respuesta deseable.

\section{Composición proximal del hidrolizado proteico}

Los valores de composición química proximal del colágeno hidrolizado de pescado se presentan en la Tabla 3 , los cuales fueron, 87 por ciento de humedad, 12,55 por ciento de proteína total, 0,32 por ciento de grasa cruda y 1 por ciento de ceniza. Por otro lado, se reportan valores químicos proximales para el hidrolizado de colágeno de pescado comercial Natural First, de 9,0 por ciento para
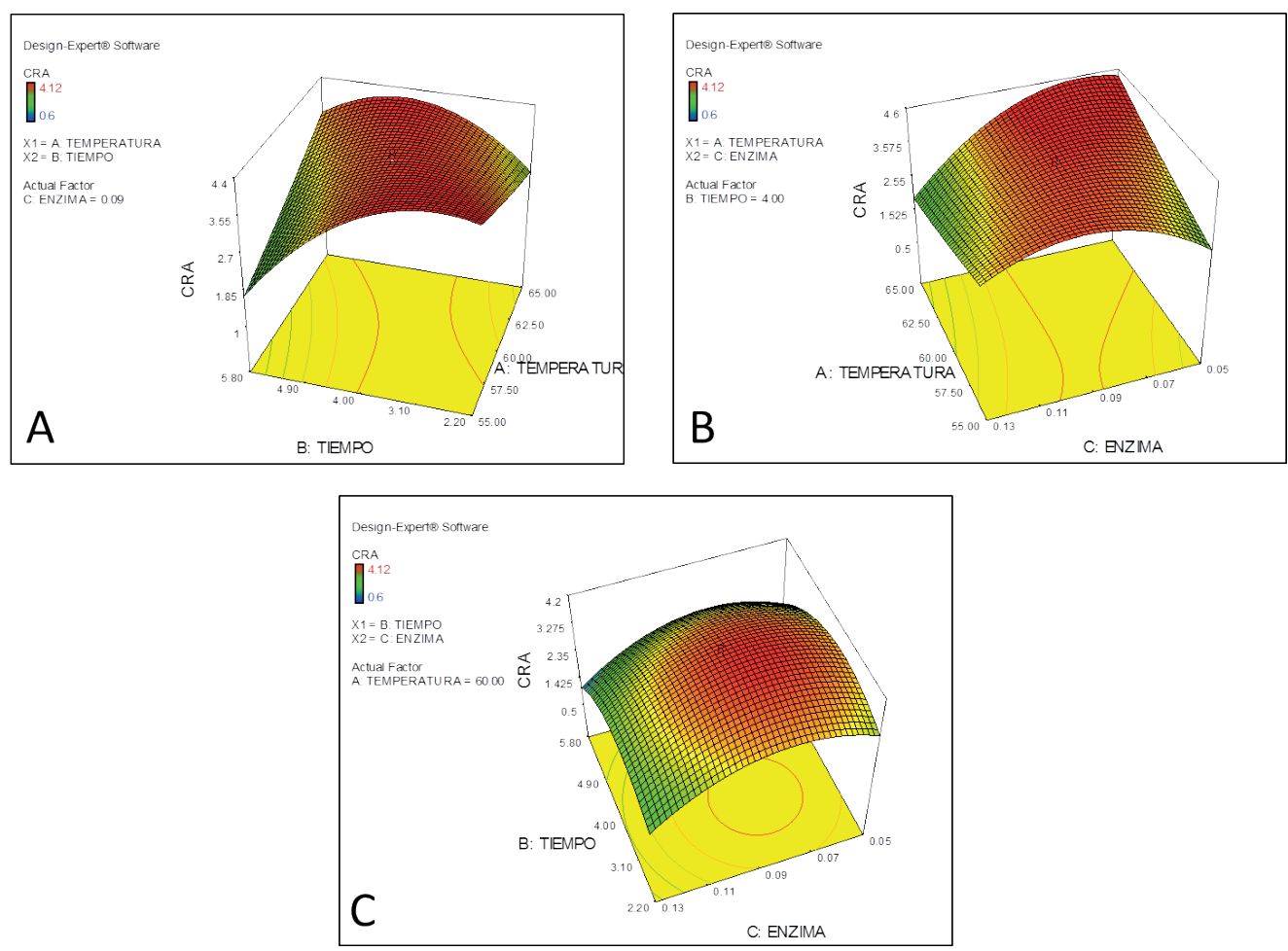

Figura 3. Superficie de respuesta para CRA del colágeno de pescado: (A) en función a las variables tiempo y temperatura; (B) en función a las variables temperatura y enzima; (C) en función a las variables tiempo y enzima

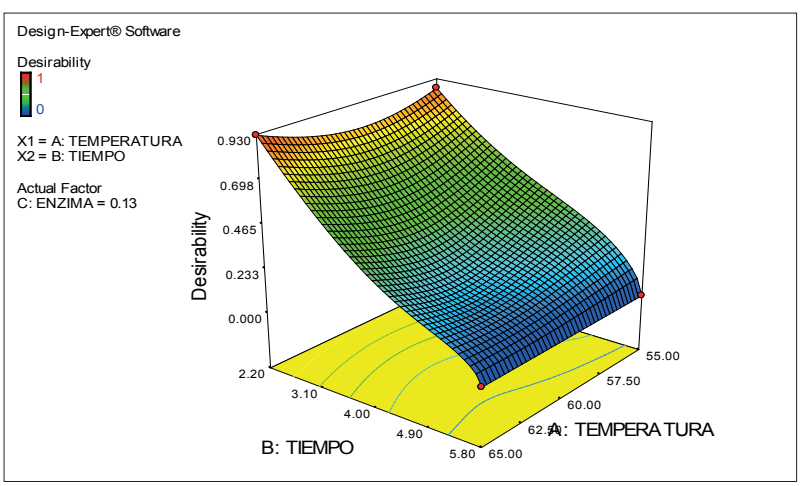

Figura 4. Superficie de respuesta de la optimización del grado de hidrólisis del colágeno de pescado purificado en función a las variables tiempo y temperatura proteína y 0,3 por ciento para grasa. Así mismo, los resultados obtenidos por Ruiz (1982), para un concentrado proteico de sardina fueron 4,72 por ciento de humedad, 0,56 por ciento de grasa, 87,57 por ciento de proteína y 2,17 por ciento de cenizas. Chávez (1980), mencionado por Pandia (2011), reporta valores para residuos de jurel, de 16,34 por ciento de proteína, 10,33 por ciento de grasa y 3,70 por ciento de ceniza. Finalmente, los valores químicos obtenidos por Pandia (2011), para residuos hidrolizados de anchoveta fueron de 13,03 por ciento de proteína, 4,35 por ciento de grasa cruda y 3,90 por ciento de ceniza. La diferencia entre estos resultados y el presente trabajo puede deberse a que estos ensayos fueron elaborados a partir de residuos frescos de pescados. 
Tabla 3. Parámetros relacionados con la composición química del colágeno hidrolizado optimizado

\begin{tabular}{lccccc}
\hline Componentes (\%) & $\begin{array}{c}\text { Colágeno hidrolizado Colágeno hidrolizado Hidrolizado de } \\
\text { de pescado }\end{array}$ & $\begin{array}{c}\text { Natural First } \\
\text { sardina }\end{array}$ & $\begin{array}{c}\text { Hidrolizado de } \\
\text { jurel }\end{array}$ & $\begin{array}{c}\text { Hidrolizado de } \\
\text { anchoveta }\end{array}$ \\
& (a) & (b) & (c) & (d) & (e) \\
\hline Humedad & 87,0 & Sin datos & 4,72 & Sin datos & Sin datos \\
Proteína total & 12,55 & 9,0 & 87,57 & 16,34 & 13,03 \\
Grasa cruda & 0,32 & 0,3 & 0,56 & 10,33 & 4,35 \\
Ceniza & 1 & Sin datos & 2,17 & 3,70 & 3,90 \\
\hline
\end{tabular}

Fuente:

(a) Resultados del presente estudio, Rodríguez (2015)

(b) Anónimo

(c) Ruiz (1982)

(d) Chávez (1980)

(e) Pandia (2011)

\section{Estimación del grado de hidrólisis}

El valor del GH para la presente investigación resultó ser 12,59 por ciento $\pm 0,45$. Pandia (2011), reporta que con un GH de 15 por ciento, obtiene mejores resultados sobre la capacidad de retención de agua y solubilidad de nitrógeno. Así mismo, Camacho et al., (2007), reporta que con un GH de 10,5 por ciento, obtiene valores óptimos para CRA, solubilidad de nitrógeno y capacidad emulsificante, concluyendo que la hidrólisis enzimática permite la formación de nuevos productos con propiedades funcionales superiores a las de la materia prima original sin hidrolizar, a excepcion de la capacidad de absorber grasas, que resulta disminuída al aumentar el GH, por el contrario los valores de absorción de agua, solubilidad y capacidad espumante se incrementarán al aumentar el $\mathrm{GH}$.

\section{Propiedades funcionales}

Los valores obtenidos en el presente estudio para la capacidad de retención de agua fue de $0,4 \mathrm{ml} \mathrm{H}_{2} \mathrm{O} / \mathrm{g}$ m para el grado de hidrólisis de 12,5 por ciento, estos resultados difieren de los reportados por Pandia (2011), quien evaluó restos frescos de anchoveta (Engraulis ringens) hidrolizada, obteniendo valores de $2,1 \mathrm{ml} \mathrm{H_{2 }} \mathrm{O} / \mathrm{g}$

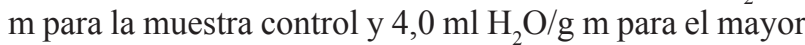
porcentaje de GH (15 por ciento). Así mismo, Camacho et al. (2007), reportó valores de 4,0 $\mathrm{ml} \mathrm{H}_{2} \mathrm{O} / \mathrm{g} \mathrm{m}$, para un GH de 10,5 por ciento de hidrolizados de pescado de caribe colorado y un $1,2 \mathrm{ml} \mathrm{H}_{2} \mathrm{O} / \mathrm{g} \mathrm{m}$, para la muestra sin hidrolizar. Estas diferencias pueden deberse a que la muestra de colágeno de pescado hidrolizada ya poseía una transformación previa antes de ejecutarse la hidrólisis enzimática en el laboratorio. La capacidad de absorber agua es de gran utilidad en la definición del uso potencial de las proteínas modificadas, principalmente si van a ser empleadas en sistemas donde se requiera una buena interacción con el agua, como es el caso de sopas, salsas, masas, productos horneados (Granito et al., 2004).

Los valores de la capacidad de emulsificación (CE) en el presente estudio fue de 1,34 $\mathrm{ml}$ aceite $/ 0,5 \mathrm{~g} \mathrm{~m}$, se asemejan a lo reportado por Camacho et al. (2007), quién evaluó la capacidad emulsificante en hidrolizados proteicos del caribe colorado (Pygocentus cariba), reportando valores de 1,41 $\mathrm{ml}$ aceite $/ 0,5 \mathrm{~g} \mathrm{~m}$ para un $\mathrm{GH}$ de 10,5 por ciento, Por otro lado, Suarez (2010), reporta valores de 1,78 ml aceite $/ 0,5 \mathrm{~g} \mathrm{~m}$, para hidrolizados de proteína de a partir de tilapia roja (Oreochromis mossambicus); mientras que Pandia (2001), reportó valores que fluctuaron entre 11,5 y $19,0 \mathrm{ml}$ aceite $/ 0,5 \mathrm{~g} \mathrm{~m}$ para el hidrolizado de anachoveta, valor máximo alcanzado con 15 por ciento de $\mathrm{GH}$, donde determina que a medida que el GH aumenta, la capacidad de emulsificación disminuye, debido a que los péptidos pequeños formados durante la hidrólisis se difunden rápidamente y se absorben en la interface aceiteagua, favoreciendo la emulsión de estos componentes, por la orientación de la parte hidrofóbica hacia la fase lipídica apolar y la región hidrofílica hacia la fase acuosa (Kristinsson y Rasco, 2000 y Jung et al., 2005).

Respecto a la capacidad de formar espuma (CFE) para el hidrolizado de colágeno de pescado purificado, se determinó un 20 por ciento de CFE, similares resultados fueron reportados por Camacho et al. (2007) para un 3,7 por ciento $\mathrm{GH}$, resultó poseer un $25,51 \% \pm 1,28 \%$ de CFE. Wasswa et al. (2008) obtuvieron tres tipos de hidrolizados a partir de la perca (Lates niloticus), carpa (Ctenopharyngodon idella) y tilapia de Nilo (Oreochromis niloticus), reportando valores de CFE que fluctuaron entre 11,9 y 28,6 por ciento. La alta capacidad espumante y estabilidad del hidrolizado puede atribuirse a un aumento de hidrofobicidad en la superficie; por otro lado, si las proteínas mantienen su estructura terciaria en la interface, pueden mantener una red intermolecular extensa (interacciones proteína-proteína) y forman películas firmes que puede dar como resultado una espuma más estable. Esta característica, le favorecería como un posible ingrediente en la formulación de alimentos que requieran formación de espuma, como en confitería, coberturas batidas y postres congelados, (Kristinsson y Rasco, 2000). Las proteínas del colágeno hidrolizadas presentaron una buena CFE. Sin embargo, la comparación de la misma se dificulta debido a que los ensayos utilizados en cada caso fueron empíricos y existen discrepancias en lo referente a los valores a considerar en el cálculo.

La capacidad de absorber aceite en función al GH de 12,59 por ciento fue de 4,54 $\mathrm{ml}$ aceite/g $\mathrm{m}$. Camacho et al. (2007), 
reportó valores de $1,36 \mathrm{ml}$ aceite/ $\mathrm{g} \mathrm{m}$, para hidrolizados proteicos del caribe colorado con un GH de 10,5 por ciento, comportamiento similar en esta propiedad fueron señalados por Kristinsson y Rasco (2000), en hidrolizados de pescado obtenidos por acción de diferentes proteasas. Estas diferencias pueden deberse al tipo de materia prima utilizada en el presente estudio, ya que previamente pasó por un proceso de extracción de colágeno en especies frescas. Por otro lado, el hidrolizado obtenido, con la capacidad de absorber grasas obtenida podría ser utilizada en la preparación de alimentos destinados a frituras, donde esta característica permitiría la retención de sabores y la disminución de la rancidez oxidativa (Kristinsson y Rasco, 2000, Granito et al., 2004).

La capacidad de gelificar para el colágeno de pescado a un pH 2, no fue posible, de igual manera ocurrió para los valores de $\mathrm{pH} 4$ y 6 , a concentraciones de 2 por ciento de colágeno. Sin embargo, si presentó capacidad de gelificación a pH de 4, 6 y 8 para las concentraciones de 4, 6 y 8 por ciento; similares resultados reportó Olivares (2009). Estos resultados obtenidos, indicaría que en soluciones de concentraciones más ácidas, la capacidad de gelificación para el colágeno hidrolizado disminuye. Una posible causa de este comportamiento, podría deberse a los tipos de enlaces presentes en la proteína, entre ellos los enlaces puente de hidrógeno, ya que éstos son sensibles a la variación del $\mathrm{pH}$, afectando a las proteínas en cuanto a su solubilidad, debido a que ésta se altera al modificar el punto isoeléctrico de las mismas, presentándose la propiedad de gelificación (Olivares, 2009).

Por otro lado, la comparación del hidrolizado de colágeno de pescado purificado se dificulta debido a que no se encontraron ensayos en donde se evalúe la capacidad de gelificación en similares materias primas.

\section{Rendimiento del proceso}

Los rendimientos obtenidos durante la elaboración del hidrolizado de colágeno de pescado purificado se reportan en la Tabla 4. El rendimiento final fue de 59 por ciento. El mayor porcentaje de disminución de peso se dio en el proceso de centrifugado ( 75 por ciento), lo que indica que en esta etapa se pierde un 25 por ciento aproximadamente del peso de la muestra inicial. No se reportan investigaciones con materia prima similar por lo que se dificulta las discrepancias en lo referente a los valores a considerar.

Tabla 2. Rendimiento por etapas del proceso de elaboración de un hidrolizado de colágeno de pescado purificado mediante el uso de la enzima Delvolase

\begin{tabular}{lcc}
\hline Material / operación & $\begin{array}{c}\text { Cantidad } \\
(\mathrm{g})\end{array}$ & $\begin{array}{c}\text { Rendimiento } \\
(\%)\end{array}$ \\
\hline Materia prima & 9,9 & 100 \\
Centrifugado & 7,5 & 75 \\
Filtrado & 5,9 & 79 \\
Hidrolizado proteico final promedio & 59 \\
\hline
\end{tabular}

\section{Conclusiones}

El hidrolizado purificado de colágeno de pescado se obtuvo empleando la enzima Delvolase, donde el proceso de hidrólisis fue influenciado por la temperatura, tiempo y concentración de la enzima en diversas combinaciones. Así mismo, el flujo de proceso constó de 7 etapas: preparación de la muestra, hidrólisis $\left(55-65^{\circ} \mathrm{C}, 2-6 \mathrm{~h}\right.$, $0.03-0.15 \%)$, inactivación de la enzima $\left(90{ }^{\circ} \mathrm{C}\right.$ por 15 $\mathrm{min}$ ), enfriado (ambiente por $5 \mathrm{~min}$ ), centrifugado (3000 rpm por $15 \mathrm{~min}$ ), envasado y almacenado. La severidad del tratamiento térmico, la concentración de enzima y el tiempo de reacción influenciaron en el grado de hidrólisis, encontrándose una relación directa con la calidad de las propiedades funcionales del hidrolizado, determinándose el grado de hidrólisis óptimo en 12.5 por ciento.

El producto final fue de color beige y olor neutro, con proteína de 12,55 por ciento, grasa de 0,32 g/100 g muestra, ceniza de 1 por ciento todo esto en base húmeda. Los resultados de las propiedades tecno-funcionales fueron, $0,4\left(\mathrm{ml} \mathrm{H}_{2} \mathrm{O} / \mathrm{g} \mathrm{m}\right)$ para la capacidad de retención de agua, 1,342 ( $\mathrm{ml}$ aceite/0,5 $\mathrm{g} \mathrm{m}$ ) para la capacidad de emulsificación, 20 por ciento para la formación de espuma, 4,54 ( $\mathrm{ml}$ aceite/ g m) para la absorción de aceite; para la capacidad gelificante se reportaron valores positivos en rangos de 4 a 8 por ciento de concentración de colágeno y de 4 a 8 de pH, así mismo, para un 2 por ciento de concentrado de colágeno a un $\mathrm{pH}$ de 8 . Finalmente, el rendimiento final del hidrolizado fue de 59 por ciento.

\section{Literatura citada}

ANFACO. 2012 Guía para el aprovechamiento de los subproductos de pescado para la obtención de productos funcionales y bioactivos. Centro Técnico Nacional de Conservación de Productos de la Pesca y Acuicultura - Ministerio de Agricultura, Alimentación y Medio ambiente. España. Ubicación en línea: http://www.magrama.gob.es/es/pesca/temas/ calidadseguridadalimentaria/06-Guia_Subproductos_ tcm7-248616_tcm7-320453.pdf .

ANÓNIMO. Colágeno hidrolizado Laffort. Ficha técnica.

Ubicación en línea:

http://www.doc4net.es/doc/3620309474472?cn - http:// www.laffort.com/en/products/fining/202.

AOAC (1990). Official Methods of Analysis. 15 $5^{\text {th }}$ ed. Association of Official Analytical Chemists, Washintong D.C. 1298 p.

Camacho D.; Moreno, M.; García, D.; Medina, C. y Sildovaras, A. 2007. Caracterización de un hidrolizado proteico enzimático obtenido del pez caribe colorado (Pygocentrus cariba) Humboldt, 1821). Interciencia: 32, 3 PRISMA; 188 - $194 \mathrm{p}$

Castañón, M. 2009. Efecto de la adición de hidrolizados de levadura Saccharomyces cerevisiae en la obtención de leches acidificadas. Tesis ing. de alimentos. Universidad de Chile, Santiago. 64 p. 


\section{CODEX ENOLÓGICO INTERNACIONAL.} PRODUCTOS ENOLÓGICOS. 2009.

Ubicación en línea: http://www.oiv.int/oiv/info/ esspecificationproduit?lang=es.

Dumay, J.; Barthomeuf, C. y Berg J-P. 2004. How Enzymes May Be Helpful for Upgrading Fish By-Products: Enhancement of Fat Extraction. Journal of Aquatic Food Product Technology, Vol. 13(2), 16 p.

FAO. 1998. El pescado fresco: su calidad y cambios de su calidad. FAO documento técnico de pesca. No. 348. Ubicación en línea: http://www.fao.org/DOCREP/ V7180/v7180s05.htm.

Fernández, A. 2001. Elaboración de un hidrolizado de residuos de pescado por fermentación en sustrato sólido con hongos filamentosos. Tesis Mg Sc. Tecnología de Alimentos. UNALM, Lima $130 \mathrm{p}$.

Gornall, A. G.; C. S. Bardwill and M. M. David. 1949. Determination of serum proteins by means of biuret reaction. J. Biol. Chem., 177: 751-766.

Gutiérrez, H.; De La Vara, R. 2012. Análisis y diseños de experimentos. Tercera edición. México. Mc Graw Hill Interamericana. $489 \mathrm{p}$.

Granito M.; Guerra, M.; Torres A.; Guinand, J. 2004. Efecto del procesamiento sobre las propiedades funcionales de Vigna sinensis. Interciencia 29: 521-526 p.

Hidalgo, L. 2014. Implementación de la propuesta de mejoramiento del proceso de producción de BIOCOL $\mathrm{P}$, basada en la optimización de recursos de la empresa Deltagen Ecuador S.A. Tesis Msc. en ingenieria industrial y productividad. Escuela Politécnica nacional. Quito, Ecuador. 211 p.

Jung S.; Murphy, P.; Johnson, LA. 2005. Physicochemical and functional properties of soy protein subtrates modified by low levels of protease hydrolysis. J. Food Sci. 70: $180-187$ p.

Kristinsson, H. G.; Rasco, B. A. 2000. Fish protein hidrolisates: Production, biochemical and functional properties. Critical Rev. In food Sci. And Nutrition 40 (1): 43-81 p.

Lin, M. J.; Humbert, E. S. and Sosulski, F. W. (1974). Certain functional properties of sunflower meal products. J, Food Sci. 39:368.

Mandeville, P. 2008. Tamaño de la muestra para modelos lineales. Ciencia UANL/Vol XII, No. 3; 351 - 355 p.

Olivares, J. 2009. Propiedades funcionales del huevo de avestruz y su aplicación en algunos productos alimenticios. Tesis maestría en ciencias de alimentos. Instituto politécnico Nacional. México. 149 p.

Pandia, S. 2011. Obtención y Evaluación de las Propiedades Funcionales de un Hidrolizado Proteico Obtenido a Partir de Anchoveta (Engraulis ringens). Tesis Ingeniero Pesquero. UNALM. Lima. 102 p.

Rodríguez-Ambríz, S.; Martínez-Ayala, A.; Millán, F. and Dávila-Ortíz, G. 2005. Composition and funtional properties of Lupinus campestris protein isolates. Plant
Food for Human Nutrition, 60, 99-107.

Suárez C. (2010). Obtencion de hidrolizado de proteína de pescado a partir de tilapia roja (oreochromis sp.). Tesis especialista en tecnología de alimentos. Universidad Nacional de Colombia. 49 p.

Wasswa, J.; Tang, J.; Gu, X. and Yuan, X. 2008. Functional properties of grass carp (Ctenopharyngodon idella), nile perch (lates niloticus) and nile tilapia (Oreochromis niloticus) skin hydrolysates. International Journal of Food Properties 11: 339 - 350.

Woo, J. W.; S. J. Yu, et al., 2008. Extraction optimization and properties of collagen from yellowfin tuna (Thunnus albacares) dorsal skin. Food Hidrocolloids 22(5): 879887.

Yu, J.; Ahmedna, M.; Goktepe, I, 2007. Peanut proteins concentrate: Production and functional properties as affected by processing. Food Chemistry, 103, 121-129. 